rheuma plus $2016 \cdot 15: 87$

DOI 10.1007/s12688-016-0097-8

Online publiziert: 24. Oktober 2016

๑) Springer-Verlag Wien 2016

\section{Burkhard Leeb}

II. Medizinische Abteilung, NOE Kompetenzzentrum für Rheumatologie, Stockerau, Österreich

\title{
Weg vom Mittelwert
}

Liebe Leserinnen und Leser,

schnell ist dieses Jahr 2016 vergangen und es erscheint noch nicht ganz klar, was diesem Jahr rheumatologisch den Stempel aufdrücken wird. Eine ähnliche Überraschung wie es die Verleihung des Literaturnobelpreises an Bob Dylan für den Schreiber dieser Zeilen war, wird es aber ziemlich sicher nicht geben.

Apropos Überraschung - eine solche war für mich, dass es eines längeren Telefonates mit einer Österreichischen Krankenkasse bedurfte, um unserem Sozialsystem durch die Verschreibung eines Biosimilars, wie ich meinte, sparen zu helfen. Die junge Kollegin, die an ihrem Schreibtisch ambitioniert versuchte die Einsparungsvorgaben zu erfüllen, war zwar nett, aber auch völlig uninformiert über Rheumatologie generell und Biosimilars überhaupt. Sie hielt sich an die offizielle Sprachregelung, die zwar nicht weitsichtig, aber einfach erscheint: Biosimilars sind dasselbe wie Generika, daher würde zwar der teurere Originator bewilligt, aber, mangels Erstattungsregelung, nicht das billigere Biosmilar. Diese Logik bleibt mir als Steuer- und Beitragszahler, damit eigentlich Teilfinanzier des Systems, unerschlossen. Übrigens, wir haben uns dann doch auf die für das System billigere Variante einigen können.

Apropos Biosimilars, vielleicht wird 2016 das Jahr der Biosimilars, immerhin wurde unter anderem ein Etanercept-Biosimilar von der FDA zugelassen, werden die Ergebnisse der NOR-SWITCH Studie präsentiert werden. Wir Österreicher können jedenfalls stolz sein einen globalen Player auf dem Biosimilar-Markt in unserem Land ansässig zu wissen. Möglicherweise wird 2016 aber auch das antiIL17- Jahr oder das Jahr der small molecules. Nicht die diesjährige Jahrestagung der Österreichischen Gesellschaft für Rheumatologie und Rehabilitation, aber die nächsten werden sich hoffentlich redlich bemühen, uns diese Frage zu beantworten.

In zunehmendem Maße bewegen sich die Medizin und auch die Rheumatologie weg von den Mittelwert gesteuerten, verallgemeinernden Therapien, die nicht direkt auf das Individuum abzielen - ja, wo es manchmal so erscheint, als wäre das Wohlbefinden des Behandlers wichtiger als das der Betroffenen. Der personalisierten Medizin gehört mit Sicherheit die Zukunft, so auch bei der häufigsten Knochenerkrankung, der Osteoporose. Birgit Harbeck und Hendrik Lehnert aus Lübeck geben in ihrer Übersicht einen Einblick in die rezenten Möglichkeiten durch neue Therapieoptionen und zeigen, wie diese nach Abwägung von Nutzen und Risiken der einzelnen Medikation den individuellen Gegebenheiten Rechnung tragen.

Die Anämie ist eine häufige Komplikation bei rheumatoider Arthritis, der unterschiedlichste Mechanismen zugrunde liegen können mit negativen Auswirkungen auf RA und Lebensqualität der PatientInnen. Wie Julia Held und Günter Weiss feststellen, findet sich diese therapiebedürftige Komplikation bei RA-PatientInnen immer noch doppelt so häufig wie in einer alters-gematchten Normalpopulation.

Schmerzen und der dringende Wunsch diese wieder los zu werden, darunter häufig Muskelschmerzen (Myalgien), führen zumeist die Patienten in die rheumatologischen Praxen, aber auch zu den ÄrztInnen für Allgemeinmedizin. Myalgien sind oft eher harmloser Natur und temporär, dennoch gibt es behandlungsbedürftige und auch gefährliche Erkrankungen, deren Leitsymptome Muskelschmerzen darstellen können. Dieser differenzialdiagnostischen Herausforderung stellt sich Jochen Zwerina in seinem beachtenswerten Übersichtsbeitrag.
Der Fallbericht in der Rubrik „Aus der Praxis“ mit dem Titel: Juckreiz, Arthralgien, pos. Autoantikörper, von Harsono T.H. Mai, Stockerau, rückt die nötige Interdisziplinarität der Rheumatologie in den Vordergrund. Wie immer an dieser Stelle möchten wir Sie herzlich dazu einladen, uns Ihre Meinung zu den Beiträgen dieser Ausgabe, wie auch zu allen rheumatologischen Themen, die Sie für relevant oder für diskussionswert halten, zu schicken. Sie können in jedem Falle auf einen fairen Review mit entsprechendem Feedback vertrauen.

Für Kritik, Hinweise, Zustimmung und für jeden anderen Beitrag sagen wir auch schon im Voraus Danke. Zu den bevorstehen Feiertagen und dem Jahreswechsel bereits jetzt die besten Wünsche.

\section{Herzlichst,}

Ihr Burkhard Leeb

\section{Korrespondenzadresse}

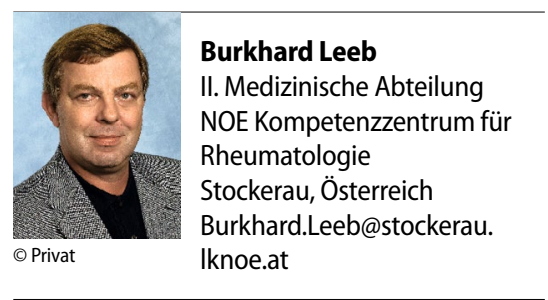

